\title{
Oportunidad de la valoración del grado de adherencia a la dieta mediterránea en pacientes con insuficiencia cardiaca en los servicios de urgencias hospitalarios
}

\section{Opportunity to assess adherence to the Mediterranean diet in patients with cardiac failure in hospital emergency services}

doi.org/10.23938/ASSN.0017

\section{P. Sánchez-Aguilera Sánchez-Paulete', M. Rubio Mellado², R. Rubio Díaz ${ }^{2}$, A. Julián-Jiménez}

\section{Sr. Director:}

Hemos leído con gran interés el reciente trabajo publicado en su revista por Miró y col en relación con la valoración del grado de adherencia a la dieta mediterránea (DMe) que tienen los pacientes con insuficiencia cardiaca (IC) ${ }^{1}$. Felicitamos a sus autores por sus resultados y conclusiones. Y más aún, por lo "sorprendente" del objetivo planteado para un estudio desarrollado en los servicios de urgencias hospitalarios (SUH), donde la falta de tiempo y la gran demanda asistencial ${ }^{2}$ conllevan habitualmente a que la única prioridad sea la atención diagnóstico-terapéutica ${ }^{3}$, y que ésta se resuelva en el menor tiempo posible, para poder dar de alta o realizar el ingreso del paciente, ya que, por lo general, hay otros enfermos urgentes esperando ser atendidos en el SUH ${ }^{2}$.

Coincidimos con los autores cuando apuntan que son los médicos de atención primaria los que tradicionalmente y oportunamente controlan la dieta de los pacientes, así como su papel en la prevención de enfermedades metabólicas o cardiovasculares $^{4,5}$. Pero esto no impide que otros especialistas implicados en el proceso y tratamiento de la insuficiencia cardiaca, como los cardiólogos ${ }^{6}$ o los médicos de urgencias ${ }^{7}$, quieran, puedan y deban aportar su experiencia para mejorar la situación global del paciente y contribuir a prevenir episodios de insuficiencia cardiaca aguda (ICA) que, sin duda, van a declinar en un deterioro de situación funcional de los pacientes y en su pronóstico. Y no es casualidad que, con frecuencia, los mismos pacientes precisarán ser atendidos y mantener un seguimiento crónico por profesionales de atención primaria y de cardiología y acumulen reiteradas visitas al SUH por episodios de ICA ${ }^{8}$.

En su trabajo, Miró y col incluyen 411 pacientes con una elevada edad media y con gran número de factores de riesgo y comorbilidad vascular, donde casi un 59\% de ellos tenían una adherencia adecuada a la DMe. Sin duda, deben constituir un subgrupo de pacientes instruidos y sensibilizados con la conveniencia de cumplir con la DMe. Pero, ¿qué pasa con el resto de la población de su edad con otras enfermedades y con la población sana? ¿Tienen éstos una adherencia similar a la DMe? Y si es así, ¿la mayor o menor adherencia a la DMe puede influir en el pronóstico y la pre-

An. Sist. Sanit. Navar. 2017; 40 (1): 159-164

1. Cardiología. Complejo Hospitalario Universitario de Toledo. España

2. Urgencias. Complejo Hospitalario Universitario de Toledo. España

Conflicto de interés: ninguno declarado Becas o ayudas: no se han recibido

\section{Correspondencia:}

Agustín Julián Jiménez

Unidad de Docencia Médica

Complejo Hospitalario Universitario de Toledo

Avda. de Barber, 30

45004 Toledo

E-mail agustinj@sescam.jccm.es 
vención secundaria de episodios de ICA en estos pacientes? ¿Y en la aparición de IC en pacientes sanos a medio-largo plazo? Creemos que todas estas preguntas quedan por demostrar en una línea de investigación muy interesante para los autores del artículo, ya que estos solo pueden llegar a la conclusión de que existe un número importante de pacientes con IC (41\%) que no siguen una DMe. Y asimismo identifican, a través del cuestionario MEDAS-14 ${ }^{1}$ distintos aspectos mejorables de la dieta en los pacientes con IC. Por ello, creemos que, de la mano de estudios como el de Miró y col, es el momento de dar un paso más e investigar la relación directa entre la adherencia (y su grado) con el pronóstico y la incidencia de episodios de ICA. En este contexto, a modo de estudio piloto, en nuestro centro hemos revisado y realizado el cuestionario MEDAS-14 por medio de una entrevista telefónica, tras consentimiento del paciente, a dos grupos de enfermos que acudieron al SUH: el primero por un episodio de ICA y el segundo (tras descartar que tuvieran el diagnóstico de IC) por un problema traumatológico accidental siempre que tuvieran
$>50$ años (Grupo A y B, respectivamente). Revisando la metodología empleada por los autores en su trabajo hemos analizado las mismas variables. La selección de los pacientes se realizó consecutivamente entre los que acudieron al SUH entre los meses de junio a septiembre de 2016 con los diagnósticos definidos y accedieron a realizar la entrevista tras el alta. A todos se les realizó en cuestionario MEDAS-14. Se consideró baja adherencia a la DMe con una puntuación de $<9$ y adecuada $\geq 9$ puntos. En la tabla 1 se muestran algunos de los resultados obtenidos. Como se puede comprobar los pacientes del grupo A son muy superponibles a los del estudio de Miró y col, en cuanto a la edad, sexo, factores de riesgo cardiovascular, situación funcional basal o tratamientos pautados. E incluso a la adherencia a la DMe y la proporción de pacientes calificados de baja o adecuada adherencia. Pero, como podemos comprobar con un perfil muy distinto al grupo de pacientes no cardiópatas que tienen una significativa menor adherencia a la DMe (solo 34\% frente al 61\%). Aunque estos resultados tienen muchas limitaciones, sí nos

Tabla 1. Características de los enfermos y comparativa entre los pacientes entrevistados

\begin{tabular}{lccc}
\hline & $\begin{array}{c}\text { Pacientes } \\
\text { Grupo A } \\
\mathbf{N = 4 4}\end{array}$ & $\begin{array}{c}\text { Pacientes } \\
\text { Grupo B } \\
\mathbf{N = \mathbf { 5 0 }}\end{array}$ & Valor p \\
\hline Edad media (DE) en años & $75,25(7,21)$ & $68,64(8,33)$ & 0,01 \\
\hline Sexo femenino: $\mathrm{n}(\%)$ & $26(59,1 \%)$ & $29(58 \%)$ & 0,025 \\
\hline Factores de riesgo': $\mathrm{n}(\%)$ & $40(90,9 \%)$ & $16(32 \%)$ & $<0,001$ \\
\hline $\begin{array}{l}\text { Situación funcional basal independiente para las actividades } \\
\text { de vida diaria (indice de Barthel = 100 puntos): } \mathrm{n}(\%)\end{array}$ & $17(38,6 \%)$ & $39(78 \%)$ & $<0,001$ \\
\hline Clase funcional NYHA I n (\%) & $11(25 \%)$ & $38(76 \%)$ & $<0,01$ \\
\hline Tratamiento con fármacos cardioprotectores ${ }^{2} \mathrm{n}(\%)$ & $38(86,4 \%)$ & $15(30 \%)$ & $<0,01$ \\
\hline Puntos totales cuestionario MEDAS-14 & $8,95(2,13)$ & $7,46(2,09)$ & 0,01 \\
Media (DE) & & & \\
\hline Baja adherencia n (\%) & $27(61,4 \%)$ & $17(34 \%)$ & $<0,001$ \\
\hline
\end{tabular}

DE: desviación estándar; DE: desviación estándar; NYHA: Clase funcional según la New York Heart Association

Grupo A: pacientes con el diagnóstico de insuficiencia cardiaca aguda (ICA) en su visita a urgencias.

Grupo B: pacientes atendidos en traumatología sin diagnóstico de cardiopatía ni ICA en urgencias

${ }^{1}$ Existencia de al menos alguno de los siguientes: hipertensión arterial, Diabetes mellitus, Dislipemia, Tabaquismo, Cardiopatía isquémica, arteriopatía periférica o enfermedad cerebrovascular

${ }^{2}$ En tratamiento al menos con uno de los siguientes: estatinas, Inhibidores del enzima convertidor de la angiotensina,

Antagonistas del receptor de la angiotensina-II, bloqueadores beta-adrenérgicos y antiagregantes. 
ponen sobre la pista que tanto en pacientes con el diagnóstico de IC (que evidentemente están más sensibilizados en la importancia de la DMe) como, sobre todo, en pacientes que nunca han sido diagnosticados de IC y, por lo tanto gozarían de buena salud, pero de malos hábitos ${ }^{9}$.

\section{BIBLIOGRAFÍA}

1. Miró Ò, Martín-SÁnchez FJ, Jacob J, Andueza JA, Herrero P, Llorens P. Valoración del grado de adherencia a la dieta mediterránea en pacientes con insuficiencia cardíaca: Estudio DIME-EAHFE. An Sist Sanit Navar 2016; 39: 261-268.

2. Tudela P, Mòdol JM. La saturación de los servicios de urgencias hospitalarios. Emergencias 2015; 27: 113-120.

3. Llorens P, Manito Lorite N, Manzano Espinosa L, Martín-SÁnchez FJ, Comín Colet J, Formiga F et al. Consenso para la mejora de la atención integral a los pacientes con insuficiencia cardiaca aguda. Emergencias 2015; 27: 245-266.

4. Madridejos Mora R, Majen Fabres L, Puig Acebal H, Sanz Latorre I, Llobet Traveset E, Arce CASAS M et al. Salut al cor/salud en el corazón: resultados del programa de educación sanitaria en salud cardiovascular de Mútua Terrassa. Aten Primaria 2014; 46: 457-463.

5. Gómez-Huelgas R, Jansen-Chaparro S, Baca-Osorio AJ, Mancera-Romero J, Tinahones FJ, BernalLÓPEZ MR. Effects of a long-term lifestyle intervention program with Mediterranean diet and exercise for the management of patients with metabolic síndrome in a primary care setting. Eur J Intern Med 2015; 26: 373-323.

6. Arós F, Estruch R. Dieta mediterránea y prevención de la enfermedad cardiovascular. Rev Esp Cardiol 2013; 66: 771-774.

7. Agudo Villa T, Álvarez-Rodríguez E, Caurel Sastre Z, Martín Martínez A, Merinero Palomares R, Álvarez Rodríguez V et al. Prevención de las complicaciones vasculares asociadas a la diabetes mellitus en los servicios de urgencias. Emergencias 2015; 27: 150-154.

8. Llorens P, Escoda R, Miró Ò, Herrero-Puente P, Martín-SÁnchez FJ, JACoB J et al. Características clínicas, terapéuticas y evolutivas de los pacientes con insuficiencia cardiaca aguda atendidos en servicios de urgencias españoles: Registro EAHFE (Epidemiology os Acute Heart Failure in Spanish Emergency Departments). Emergencias 2015; 27: 11-22.

9. Estruch R, Ros E, Salas-Salvadó J, Covas MI, Corella D, Arós F et al. Primary prevention of cardiovascular disease with a Mediterranean diet. N Engl J Med 2013; 368: 1279-1290. 



\title{
Grado de adherencia a la dieta mediterránea en pacientes con insuficiencia cardiaca en los servicios de urgencias hospitalarios
}

\section{Degree of adherence to the Mediterranean diet in patients with heart failure in hospital emergency services}

\author{
Ò. Miró ${ }^{1}$, P. Llorens ${ }^{2}$
}

\section{Sr. Director:}

En primer lugar, agradecemos el interés y las felicitaciones que nos trasladan los autores en relación a nuestro trabajo previamente publicado en la revista Anales del Sistema Sanitario de Navarra ${ }^{1}$. Se muestran sorprendidos por el hecho que especialistas en urgencias lleven a cabo una entrevista dietética de cierta complejidad y que requiere tiempo en pacientes atendidos en urgencias, pero debemos remarcar que, como se comentaba en nuestro trabajo, la entrevista se realizó telefónicamente a posteriori, y no durante la atención médica en urgencias. Ciertamente, compartimos con los autores que los servicios de urgencias, a menudo saturados de pacientes ${ }^{2}$, no son un lugar propicio para el desarrollo de acciones que sobrepasen la estricta valoración clínica y terapéutica. No obstante, también creemos que en determinados entornos, en pacientes con ciertas patologías prevalentes y con una asignación mínima de recursos pueden llevarse a cabo determinadas acciones específicas con valor añadido ${ }^{3-7}$.

En esta misma línea argumentativa, nuestro trabajo en pacientes con insuficiencia cardiaca aguda (ICA) puede constituir un ejemplo incipiente de intervención,

1. Área de Urgencias. Hospital Clínic. IDIBAPS. Universitat de Barcelona. Barcelona. España.

2. Servicio de Urgencias. Unidad de Corta Estancia y Hospitalización a Domicilio. Hospital General de Alicante. Alicante. España. especialmente si se demostrase algún efecto beneficioso de la adherencia a la dieta mediterránea (DiMe) en la prevención secundaria de nuevos episodios de ICA. De forma preliminar, y respondiendo a alguna de las cuestiones planteadas por los autores, estamos expandiendo los resultados de nuestra primera comunicación utilizando exactamente la misma aproximación metodológica $^{1}$ y realizando un seguimiento de los pacientes a 90 días. Así, hemos observado que entre 934 pacientes dados de alta tras un episodio de ICA a los que se encuestó su hábito dietético mediante el cuestionario MEDAS, la necesidad de rehospitalización a los 3 meses en los adherentes a una DiMe fue del 13,6\%, frente al $17,9 \%$ en los pacientes no adherentes (odds ratio 0,72 ; intervalo de confianza del $95 \%$ 0,51-1,03; $\mathrm{p}=0,08$ ). Por tanto, es posible que en un futuro puedan desarrollarse intervenciones dietéticas en los pacientes con ICA encaminadas a reducir las rehospitalizaciones y, tal vez, algunas de ellas puedan iniciarse en los servicios de urgencias o en unidades adscritas a ellos ${ }^{8,9}$.

Finalmente, los autores muestran datos propios en dos subgrupos de pacientes. Por lo que respecta a sus pacientes con ICA, sus hallazgos son superponibles a los nuestros procedentes de una población de

\section{Correspondencia:}

Oscar Miró

Servicio de Urgencias

Hospital Clínic

Carrer de Villarroel, 170

08036 Barcelona 
pacientes con ICA de Madrid y Barcelona, por lo que creemos que los refuerzan y sugieren que, posiblemente, sean extensibles a gran parte de la población española: alrededor del $60 \%$ de los pacientes con ICA en España pueden considerarse adherentes a la DiMe ${ }^{1}$. En cuanto a los que hacen referencia a pacientes no cardiópatas atendidos en urgencias por un problema traumatológico accidental, que podríamos considerar una aproximación a la población general, es muy relevante que su adherencia a dicha DiMe sea menor a la de los cardiópatas (del 34\% frente al 61\%). Esto debe hacernos plantear, más aún si cabe, la necesidad de transmitir hábitos de salud desde cualquier punto del sistema sanitario, servicios de urgencias incluidos, y especialmente los dietéticos, habida cuenta de que ya ha sido demostrada su capacidad de prevención primaria de eventos cardiovasculares adversos en pacientes de alto riesgo ${ }^{10}$. Y para avanzar en todos estos aspectos, creemos que la mejor manera es hacerlo de manera conjunta todos los profesionales y especialidades que actúan sobre una misma patología, en este caso, la ICA, venciendo las posibles dificultades de diversa índole que tal colaboración pueda plantear ${ }^{11-15}$.

\section{BIBLIOGRAFÍA}

1. Miró Ò, Martín-SÁnchez FJ, Jacob J, Andueza JA, Herrero P, Llorens P. Valoración del grado de adherencia a la dieta mediterránea en pacientes con insuficiencia cardíaca: Estudio DIME-EAHFE. An Sist Sanit Navar 2016; 39: 261-268.

2. Tudela P, Mòdol JM. La saturación de los servicios de urgencias hospitalarios. Emergencias 2015; 27: 113-120.

3. Fuenzalida Inostroza CI, Ferró Ricart I, Siches Cuadra C, Ambrós Ribó A, Sánchez M, Cabrera $\mathrm{J}$ et al. Una intervención educativa de enfermería al alta de urgencias disminuye complicaciones e ingresos a corto plazo en pacientes con fibrilación auricular. Emergencias 2015; 27 : 75-81.

4. Iglesias López S, Llopis García G, Yañez-Palma MC, RodríGuEZ AdRada E. Detección del paciente paliativo con insuficiencia cardiaca aguda en urgencias. An Sist Sanit Navar 2016; 39: 323-324.
5. Tomás Vecina S, Mozota Duarte J, Ortega Marcos M, Ruiz Navarro MG, Borillo V, SAN Juan GAGo L et al. Estudio sobre la reducción de eventos adversos en pacientes y problemas de bioseguridad de los profesionales derivados de la aplicación de catéteres vasculares en urgencias. Emergencias 2016; 28: 89-96.

6. Agudo Villa T, Álvarez-Rodríguez E, Caurel Sastre Z, Martín Martínez A, Merinero Palomares R, Álvarez Rodríguez V et al. Prevención de las complicaciones cardiovasculares asociadas a la diabetes mellitus en los servicios de urgencias. Emergencias 2015; 27: 150-154.

7. Valle Alonso J, Pedraza J, Lopera Lopera E, MuÑOZ MC, NAVARRO C, URBANO MM. Intervención en el servicio de urgencias para lograr un inicio y seguimiento adecuados de la anticoagulación en pacientes con fibrilación auricular. Emergencias 2015; 27: 82-86.

8. Miró Ò, Carbajosa V, Peacock WF, Llorens P, Herrero P, JACOB J et al. The effect of a shortstay unit on hospital admission and length of stay in acute heart failure: REDUCE-AHF study. Eur J Intern Med 2017; en prensa (doi: 10.1016/j.ejim.2017.01.015).

9. Carbajosa V, Martín-Sánchez FJ, Llorens P, HeRRERo P, JACOB J, AlquÉZAR A et al. Factores asociados a estancias cortas en los pacientes ingresados por insuficiencia cardiaca aguda. Emergencias 2016; 28: 366-374.

10. Estruch R, Ros E, Salas-Salvadó J, Covas Mi, Corella D, Arós F et al. Primary prevention ofcardiovascular disease with a Mediterranean diet. N Engl J Med 2013; 368: 1279-1290.

11. BuEno H. Continuidad en la investigación cardiovascular: una llamada a incrementar la colaboración en investigación entre Cardiología y la Medicina de Urgencias y Emergencias. Emergencias 2015; 27: 396-398.

12. Llorens P, Manito Lorite N, Manzano Espinosa L, Martín-SÁnchez FJ, Comín Colet J, Formiga F, et al. Consenso para la mejora de la atención integral a los pacientes con insuficiencia cardiaca aguda. Emergencias 2015; 27: 245-266.

13. MiRó O. Necesidad de colaboración en investigación entre cardiólogos y médicos de urgencias. An Sist Sanit Navar 2014; 37: 9-16.

14. García J, Mellado FJ, Rosell J. Dificultades para la colaboración en investigación entre cardiólogos y médicos de urgencias. An Sist Sanit Navar 2015; 38: 137-138.

15. MiRó O. Dificultades para la colaboración en investigación entre cardiólogos y médicos de urgencias. An Sist Sanit Navar 2015; 38:141-143. 